\title{
Intelligent Segmentation of Medical Images using Fuzzy Bitplane Thresholding
}

\author{
Z. Faizal Khan ${ }^{1}$, A. Kannan ${ }^{2}$ \\ ${ }^{1}$ Anna University Chennai, Chennai-600025, India. \\ ${ }^{2}$ Department of Information Science and Technology, Anna University Chennai, Chennai-600025, India. \\ faizalkhan_111@yahoo.co.in, kannan@annauniv.edu
}

\begin{abstract}
The performance of assessment in medical image segmentation is highly correlated with the extraction of anatomic structures from them, and the major task is how to separate the regions of interests from the background and soft tissues successfully. This paper proposes a fuzzy logic based bitplane method to automatically segment the background of images and to locate the region of interest of medical images. This segmentation algorithm consists of three steps, namely identification, rule firing, and inference. In the first step, we begin by identifying the bitplanes that represent the lungs clearly. For this purpose, the intensity value of a pixel is separated into bitplanes. In the second step, the triple signum function assigns an optimum threshold based on the grayscale values for the anatomical structure present in the medical images. Fuzzy rules are formed based on the available bitplanes to form the membership table and are stored in a knowledge base. Finally, rules are fired to assign final segmentation values through the inference process. The proposed new metrics are used to measure the accuracy of the segmentation method. From the analysis, it is observed that the proposed metrics are more suitable for the estimation of segmentation accuracy. The results obtained from this work show that the proposed method performs segmentation effectively for the different classes of medical images.
\end{abstract}

Keywords: fuzzy logic, bitplane method, computed tomography (CT), segmentation, unsupervised method.

\section{INTRODUCTION}

$\mathrm{I}_{\mathrm{r} w \boldsymbol{n}}^{\mathrm{N}}$ MAGE SEGMENTATION is one of the important tasks for image understanding and analysis. Segmentation partitions an image into a set of different regions. Each segmented object is homogeneous with respect to certain intensity characteristics like color and texture. Segmentation of medical imaging is useful for extracting information about the status of different tissues, different organs and other parts of the body. The accuracy and higher decision confidence value of any abnormality identification system relies mostly on an efficient and effective segmentation technique. Therefore, it is very important to achieve an effective performance of the system to provide them with entire and perfectly complete segmentation technique. The existing medical image segmentation techniques generally have limitations since most researchers are focusing only on segmenting the images of single organs. However, it is necessary to have a generic segmentation algorithm which can segment the images of different organs of the human body.

In the recent literature [6], a number of approaches have been proposed for segmenting medical images effectively. However, most of the existing methods have used thresholding [1, 2, 910 and 11] based methods for segmentation. Moreover, there are many methods like Watershed Transform [3, 7], Region growing algorithm [4], Model-based methods, edge-tracking approach [17], 3D modelling [22], and Multiscale morphological segmentation techniques $[18,5]$.

\subsection{Intensity based medical image segmentation methods}

The intensity based methods depend mostly on the brightness constant called intensity (threshold) value of the pixels in the original image.
A combination of background-removal operator and iterative gray level thresholding was proposed [8] for segmenting medical images. In their work, the background was not eliminated well due to the presence of noise. A novel method for segmenting the lung regions by combining a threshold-based method with adaptive border marching was given in [9]. They have analyzed their algorithm for 20 slices. The metrics used to evaluate their segmentation accuracy is volume-based over- and under-segmentation. They got an over- and under-segmentation accuracy of $0.43 \%$ and $1.63 \%$, respectively, for 20 slices.

A new threshold based method for segmenting the lungs from diseased CT lung images by selecting an optimal threshold for a given image by comparing the curvature of the lung boundary along with the ribs was proposed in [10]. The threshold value is checked until the curvature of the ribs and the curvature of the lung boundary are closely matched with one another. The performance of threshold-based lung segmentation algorithms in a large-scale robustness analysis is illustrated in [11]. They have applied their proposed method on a set of 2768 chest CT scans which consists of 2292 slices. The slices were taken under various abnormalities and scanning positions. Automatic thresholdbased lung segmentation was performed and visually checked in all the slices. Average error rate of $4.4 \%$ was obtained in 121 scans.

A combination of several image processing techniques with support vector machine (SVM) was proposed in [12] for effective segmentation vessels in retina images. Line detector is used to identify the presence of thin vessels in the retina images. A combination of vessel direction and the eigenvector of the Hessian matrix are used in order to obtain a segmented vessel tree which results in accuracy of $93.28 \%$ of segmentation. 
A combination of the radial projection and support vector machine (SVM) is proposed in [21] for the process of segmenting vessels present in the CT retina images. In their work, the blood vessels are located using radial projections. The SVM classifier is used in a semi-supervised selftraining to extract the major structure of vessels. An average accuracy of 0.7410 is obtained for a set of datasets taken from the DRIVE database.

An effective retinal vessel segmentation technique based on supervised classification using an ensemble classifier of boosted and bagged decision trees was proposed in [26]. A novel neural network based methodology for the segmentation of retinal vessels was presented in [25]. The neural network is trained in only one database which proves to be effective and robust in segmenting the vessels present in retina images. An average accuracy of 0.9452 is obtained for the set of database images.

An improved watershed transform using prior information was proposed in [3], which segments multiple medical images including knee cartilage and gray/white matters. An automated multi-organ segmentation method for 3D abdominal CT images based on a spatially-divided probabilistic atlas was proposed in [19]. Their proposed method was evaluated using 100 abdominal CT images with manually created ground truth data. Experimental results revealed that their algorithm can segment the liver, spleen, pancreas, and kidneys with an accuracy rate of $95.1 \%$, $91.4 \%, 69.1 \%$, and $90.1 \%$, respectively. An automatic missing organ detection scheme was explained in [20] by combining the abnormalities present in the post-surgical organ motion and organ-specific intensity homogeneity, along with the atlas-based multi-organ detection. Their proposed method was validated with 44 abdominal CT scans resulting in $93.3 \%$ accuracy for detecting the multiple organs.

From the works found in the literature, it has been observed that most of the existing works were proposed for segmenting only single organs in medical images. Therefore, it is necessary to propose a new and efficient technique to segment the multiple organs in medical images and to measure the accuracy of segmentation. In such a scenario, the existing methods are not suitable to measure the segmentation accuracy. Therefore, it is necessary to propose a new metrics for multi-organ segmentation algorithm.

\section{FUZZY BITPLANE THRESHOLDING}

In this paper, a fuzzy logic with bitplane thresholding has been proposed for effective segmentation of medical images. This proposed method is useful for making effective decisions under uncertainty. In addition, new metrics have been proposed in this method for effective measurement of segmentation accuracy.

\subsection{Overview of the proposed method}

The FLBP method is a combination of bitplane processing of the image and fuzzy logic applied to each pixel. For implementing the FLBP method:

a) The grayscale values in the $\mathrm{CT}$ image are considered to decide the threshold value. b) The grayscale image is separated into 8 monochrome images in order to obtain the bit values from each bitplane.

c) Eight features are formed at each pixel. They are F8, F7, F6, F5, F4, F3, F2, and F1. The numbers in the feature variables indicate the bitplane.

d) Three signum membership functions are used to form a fuzzy membership table in order to assign a bit value ' 0 ' or ' 1 ' to the bitplanes.

e) Inference is carried out for the foreground and background based on the bit values assigned using membership table.

f) Defuzzification is performed with maximum number ' 1 ' or ' 0 ' in a 3 X 3 window considering foreground and background bit values of each pixel to decide the final segmentation.

g) Regions of interest are separated from its original CT images.

The proposed system is based on grayscale processing. Fuzzy rules are developed and used for determining the optimum segmentation of anatomic structures from the background of medical images. In this work, lung and diabetic retinopathy images are taken as example to prove the suitability of the proposed FLBT in segmenting medical images. The diabetic retinopathy images are taken using a FUNDUS camera. The lung images were taken from Lung Image Database Consortium (LIDC) database. These images are taken as example since both are grayscale images.

\subsection{Fuzzification}

The process of transforming the values from one crisp set into fuzzy set members for qualitative representation is called fuzzification. In this work, the transformation process is performed by the formation of 8 bitplanes, such as Bitplane-1, Bitplane 2, Bitplane 3,.....Bitplane 8. Each bitplane of the image contains 1or 0 . Hence, each bitplane is a monochrome image. By observing each bitplane, the gradient of the boundary of image is calculated and a decision is made whether segmentation is required at such gradients. The degree of membership of each object in the image is computed on its association to each bitplane.

\subsection{Feature extraction}

Feature extraction is a process of extracting representative values that will uniquely distinguish one image from a group of images. In this work, features extracted from lungs are different from the features of other parts of lungs. Many methodologies are available for extracting features of various objects in the lung image. These features are usually normalized values. The 8 features obtained at each pixel are grouped into two categories, namely Foreground (FG) and Background (BG) features as shown in Table 1. Features (F8, F7, F6, F5) belong to the background and features (F4, F3, F2, F1) belong to the foreground. 
Table 1. Feature variables corresponding to each bitplane.

\begin{tabular}{|c|c|c|}
\hline Bitplane-8 & F8 & \multirow{4}{*}{ 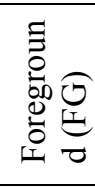 } \\
\hline Bitplane-7 & F7 & \\
\hline Bitplane-6 & F6 & \\
\hline Bitplane-5 & F5 & \\
\hline Bitplane-4 & F4 & \multirow{4}{*}{ 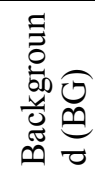 } \\
\hline Bitplane-3 & $\mathrm{F} 3$ & \\
\hline Bitplane-2 & $\mathrm{F} 2$ & \\
\hline Bitplane-1 & F1 & \\
\hline
\end{tabular}

\subsection{Membership table formation}

Membership degree can be expressed by a mathematical function $\mu_{\mathrm{A}}\left(\mathrm{x}_{1}\right)$ that assigns each element in the set a membership degree between ' 0 ' and ' 1 '. Let $\mathrm{X}$ be the universe of discourse and $\mathrm{x}_{\mathrm{i}}$ is an element of $\mathrm{X}$. A fuzzy set $\mathrm{A}$ in $\mathrm{X}$ can be defined as [14]

$$
\mathrm{A}=\left\{x_{i}, \mu_{\mathrm{A}}\left(x_{i}\right) \mid x_{i} \in X\right\}
$$

The signum function [15] (Sig) is used for modeling the membership degrees. This degree of function is suitable for representing the white and black pixels. In this proposed work, we are focusing on segmentation by using bits. In such case, we get either ' 0 ' or ' 1 ' rather than getting any intermediate outputs. Hence, we are choosing only signum function.

\subsubsection{Triple signum function}

In this paper, we propose a new signum function called triple signum function that outputs the sign of a real number. This function outputs any one value from the three values ($1,0,1)$, when a real number is given as input to the proposed triple signum function. In order to propose this function, the existing signum function has been modified in this work for suiting our segmentation algorithm by outputting the bit values $(1,0)$ and hence the outputs of the signum function are $(1,0)$. This function can be also called as step function since only $(1,0)$ are obtained.

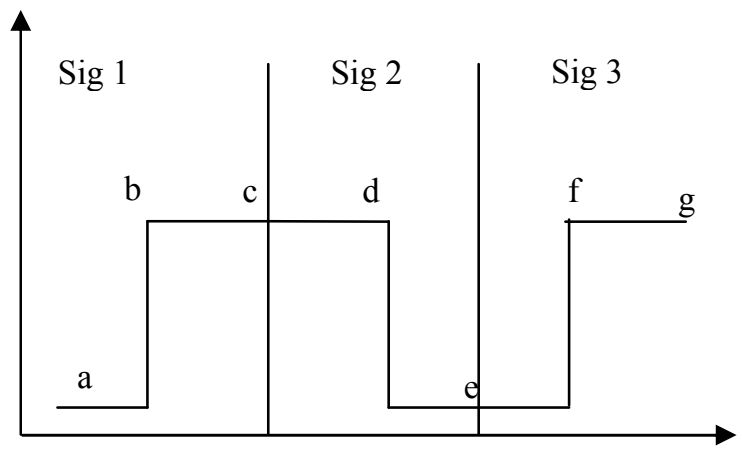

Fig.1. Triple signum function.

Three signum functions, sig1, sig2, and sig3 shown in Fig.1., are used in this work since three interval levels of thresholds are used. The width of signum 1 is "a-b-c". The position of " $b$ " can be moved towards "a" or towards "c". The width of signum function 2 is "c-d-e". The position of "d" can be shifted toward "c" or towards "e". Similarly, the width of signum function 3 is "e-f-g". The position of " $\mathrm{f}$ " can be moved towards "e" or towards " $\mathrm{g}$ ".

Shifting the position of $b, d$, and $f$ is based on the segmentation accuracy obtained. The sig2 and sig3 functions are suitable for representing the set of FG bits and is defined as

$$
\begin{aligned}
\mathrm{A} & =\mu_{\mathrm{A}-\operatorname{sig}(\mathrm{x})}=\operatorname{sig}(x, a, b, c) \\
& = \begin{cases}1 & b \leq x \leq c \\
0 & c \leq x \leq d \\
1 & x \geq d\end{cases}
\end{aligned}
$$

The sig1 and sig2 function is suitable for representing the set of BG bits and is defined as

$$
\begin{aligned}
& \mathrm{A}=\mu_{\mathrm{A}-\operatorname{sig}(\mathrm{y})}=\operatorname{sig}(x, a, b) \\
& \mathrm{A}= \begin{cases}0 & x \leq a \\
1 & a \leq x \leq b\end{cases}
\end{aligned}
$$

Membership table helps to assign the degree of membership value for the intensity values of different pixels. The membership table is similar to a look up table. The membership table formation is required to associate the input information into output information. The Fuzzy rules for assigning values from membership table are as follows:

\begin{tabular}{|l|l|}
\hline Fuzzy rule 1 & $\begin{array}{l}\text { IF F8 } \geq \frac{2}{3} \text { F7 then F8 }=1 \\
\text { Else V8 }=0 \\
\text { ENDIF }\end{array}$ \\
\hline Fuzzy Rule 2 & $\begin{array}{l}\text { IF F7 } \geq \frac{2}{3} \text { F6 then F7 }=1 \\
\text { Else F7 }=0 \\
\text { ENDIF }\end{array}$ \\
\hline Fuzzy Rule 3 & $\begin{array}{l}\text { IF F6 } \geq \frac{2}{3} \text { F5 then F6 }=1 \\
\text { Else F6 }=0 \\
\text { ENDIF }\end{array}$ \\
\hline Fuzzy Rule 4 & $\begin{array}{l}\text { IF F5 } \geq 10 \text { thenV5 }=1 \\
\text { Else V5 }=0 \\
\text { ENDIF }\end{array}$ \\
\hline Fuzzy Rule 5 & $\begin{array}{l}\text { IF F4 } \geq 5 \text { then F4 }=1 \\
\text { Else F4 }=0 \\
\text { ENDIF }\end{array}$ \\
\hline Fuzzy Rule 6 & $\begin{array}{l}\text { IF F3 } \geq 5 \text { then } \\
\text { F3 }=1 \\
\text { Else F3 }=0 \\
\text { ENDIF }\end{array}$ \\
\hline Fuzzy Rule 7 & $\begin{array}{l}\text { IF F2 } \geq 2 \text { then F2 }=1 \\
\text { Else V2 }=0 \\
\text { ENDIF }\end{array}$ \\
\hline
\end{tabular}




\subsection{Defuzzification}

Defuzzification is the process of combining the outputs of the inference for each pixel to a final segmented output that provides a segmented foreground against the background of the medical image. There are at least 15 different defuzzification methods. Moreover, we have used the "Maximum" defuzzification method [13]. Due to its generality, maximum refers to the total number of occurrence of either ' 1 ' or ' 0 '. The maximum $\mathrm{M}$ of the mean can be calculated as follows:

$$
\mathrm{A}=\sum_{i=1}^{n} \frac{\mathcal{u}_{i}}{n}
$$

Where $\mathrm{n}=1$ to 9 and $\mathrm{u}_{\mathrm{i}}$ is the location of bit value 1 .

Output of the fuzzy rules is further defuzzified in order to form a crisp value $\boldsymbol{\theta}_{k}$ to the Foreground and Background. The equation for obtaining a (crisp) bit value for Foreground is as follows:

$$
\theta_{F G}=\frac{\sum_{i=1}^{n} \delta^{n} \mu_{(A-s i g(x))}}{\sum_{i=1}^{n} \delta^{n}}
$$

Similarly, the equation obtaining a (crisp) bit value for background is as follows:

$$
\theta_{B G}=\frac{\sum_{i=1}^{n} \delta^{n} \mu_{(A-s i g(y))}}{\sum_{i=1}^{n} \delta^{n}}
$$

Where

$$
\delta^{\mathrm{n}}=\sum\{\delta(\text { rule } 1)+\delta(\text { rule } 2) \ldots \ldots . .+\delta(\text { rule } \mathrm{n})\}
$$

$\theta_{B G}$ and $\theta_{F G}$ are the crisp values which are assigned to the Foreground and Background, $\mathrm{n}$ is the rule number and $B^{\text {n }}$ is the membership function.

$$
\mathrm{f}_{\mathrm{s}}=\left\{\begin{array}{ll}
1 & \text { if } \\
0 & \text { else }
\end{array} \theta_{F G}=1\right.
$$

Final segmented region $f_{s}$ is obtained from the above equation through the crisp values ' 0 ' and ' 1 '.

\section{EXPERIMENTAL EVALUATION}

The accuracy of segmentation has been evaluated in this work in order to assess the performance of proposed algorithm in segmenting two different medical images. In this section, we present the results of the proposed method qualitatively and quantitatively through image display and experiment measurements. It is easier to evaluate the segmentation accuracy of a method if the segmented object does not have any other smaller objects when compared to the same location of the original image based on counting the number of pixels in the original image. The following two categories of medical images, namely lung images and Eye fundus images, are utilized in the experimentation.

\subsection{Existing metrics to measure the segmentation accuracy}

The metrics $A_{s}$ defined in [16] is used to measure the accuracy of medical images. In our method, the segmentation accuracy $A_{\text {pixel }}$ is computed using the formula.

$$
A_{\text {pixel }}(\%)=\frac{N_{c}}{T_{p}} X 100
$$

Where $A_{\text {pixel }}$ is the segmentation accuracy. $N_{c}$ is the number of correctly segmented pixels in the segmented image and $T_{p}$ is the total number of pixels corresponding to the unsegmented image.

Fig.2. and Fig.3. give the results of the proposed FLBT technique when applied on the lung and retina data sets. The images shown in the first column are original images and the images shown in the second column are the segmented images obtained by applying the proposed algorithm. From these figures, it is observed that the proposed system produces much smoother results than the other schemes that have been used earlier for segmenting multiple medical images. It is also observed that almost all the existing techniques do not provide better results where segmenting multiple organs is concerned in which there are overlaps of intensities in medical images. Table 2. and 3. show the segmentation accuracy results obtained by segmenting the medical images and are compared using the accuracy metrics.

Table 2. Segmentation accuracy for each lung image using metrics 1 .

\begin{tabular}{|l|l|c|l|}
\hline Image & $\mathrm{N}_{\mathrm{C}}$ & $\mathrm{T}_{\mathrm{P}}$ (Ground truth) & $\mathrm{A}_{\text {pixel }}(\%)$ \\
\hline Image 1 & 40232 & 41882 & 96.06 \\
\hline Image 2 & 38002 & 38555 & 98.56 \\
\hline Image 3 & 31232 & 31896 & 97.85 \\
\hline Image 4 & 41362 & 42372 & 97.61 \\
\hline
\end{tabular}

Table 3. Segmentation accuracy for each retina image using metrics-1.

\begin{tabular}{|l|c|c|l|}
\hline \multicolumn{1}{|c|}{ Image } & $\mathrm{N}_{\mathrm{C}}$ & $\mathrm{T}_{\mathrm{P}}$ (Ground truth) & $\mathrm{A}_{\text {pixel }}(\%)$ \\
\hline Image 1 & 1296 & 1356 & 95.57 \\
\hline Image 2 & 1163 & 1237 & 94.01 \\
\hline Image 3 & 1223 & 1298 & 94.22 \\
\hline Image 4 & 1160 & 1249 & 92.87 \\
\hline
\end{tabular}



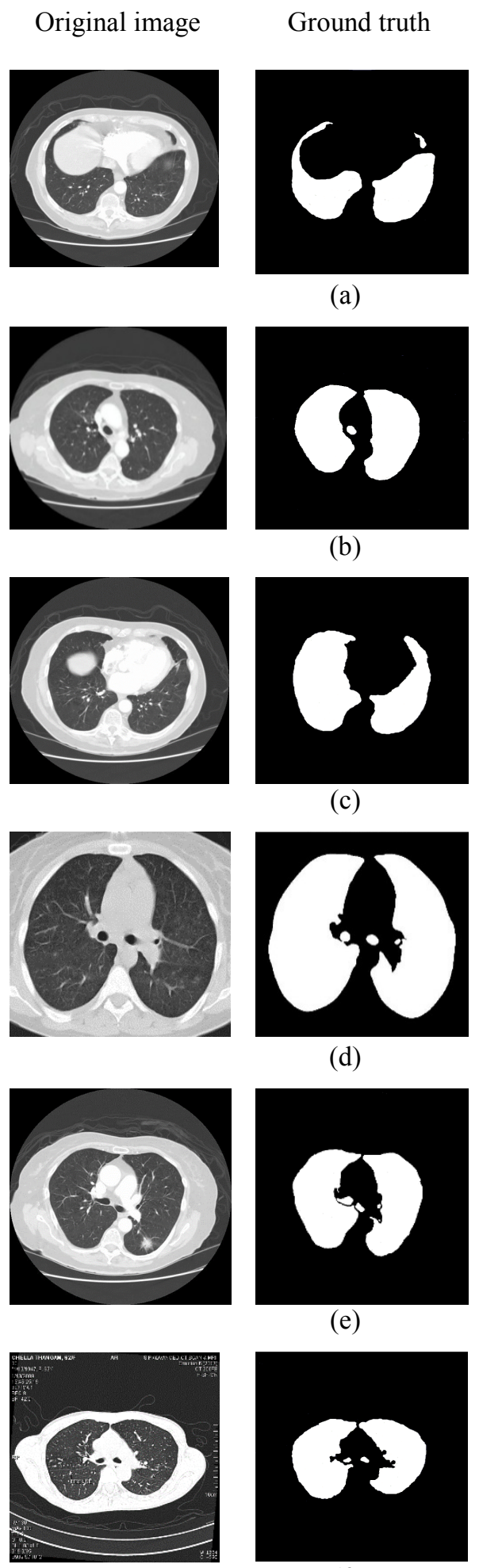

(f)
Segmented

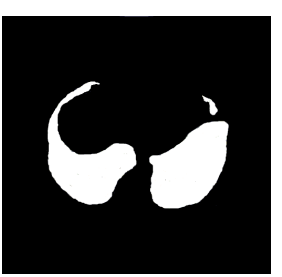

(a)

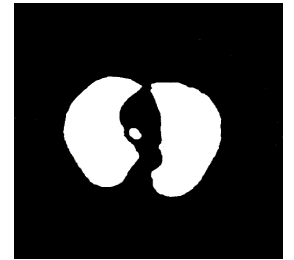

(b)

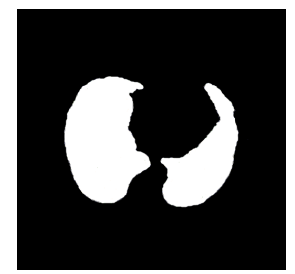

(c)

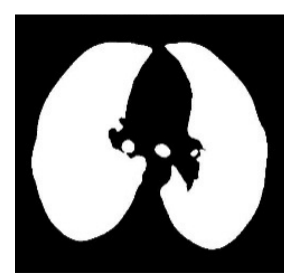

(d)

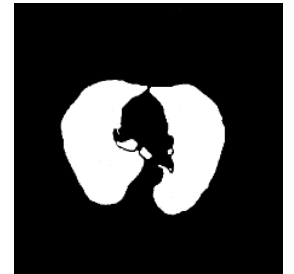

(e)
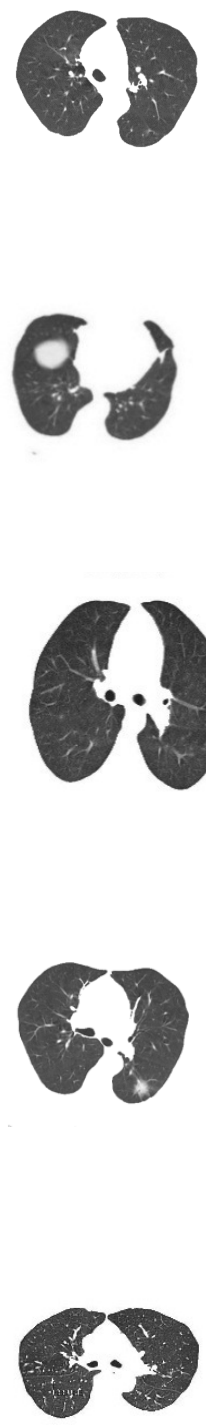

Fig.2. (a-f) Segmentation results of the proposed segmentation algorithm for lung images.

Original image
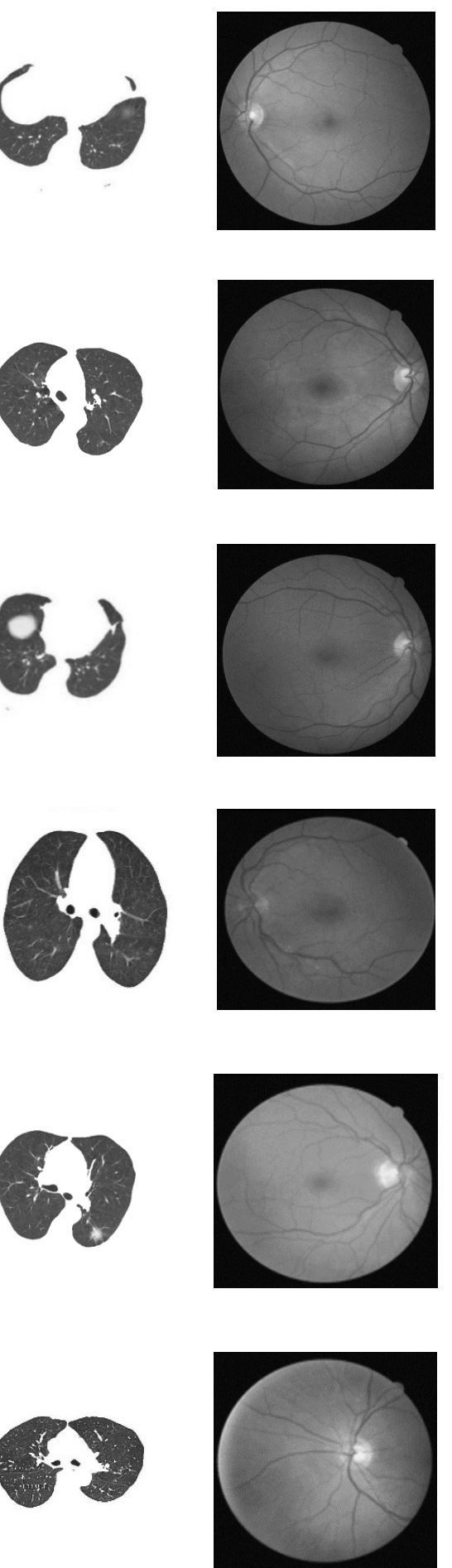

Fig.3. (a-f) Segmentation results of the proposed segmentation algorithm for retina images.
Ground truth Segmented
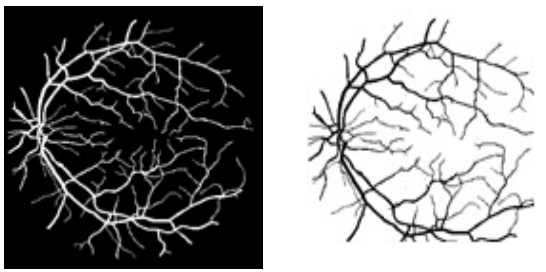

(a)
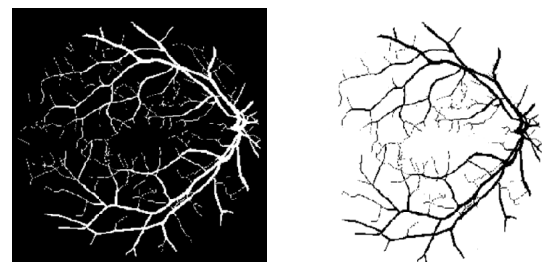

(b)
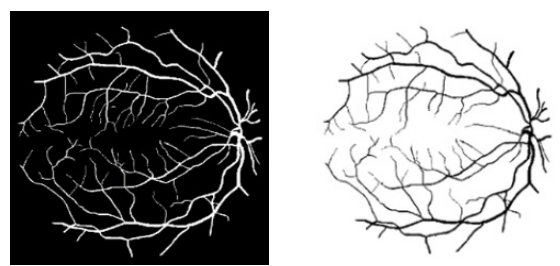

(c)
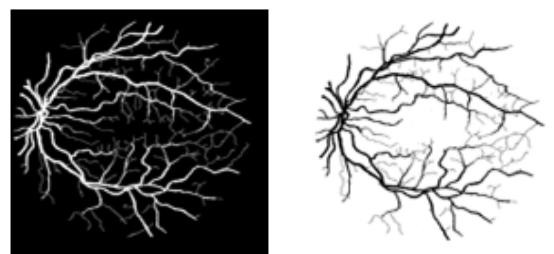

(d)
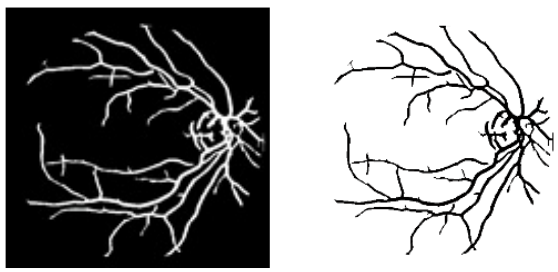

(e)
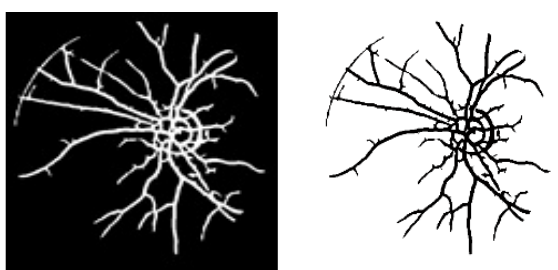

(f) 


\subsection{Proposed measures to measure the segmentation accuracy}

In this paper, we proposed a metrics to evaluate the segmentation accuracy by using the region properties of images. We define the segmentation accuracy $A_{\text {prop }}$ using the following equation to evaluate the quality of the segmented lungs:

$$
A_{\text {prop }}=\frac{S_{\text {prop }}}{O_{\text {prop }}} \times 100
$$

In addition, we proposed two new metrics, namely $\mathrm{S}_{\text {prop }}$ and $\mathrm{O}_{\text {prop }}$ which are defined as follows:

$$
\begin{aligned}
& S_{\text {Prop }}=(\text { Solidity }+ \text { Area }+ \text { Perimeter }) \\
& O_{\text {Prop }}=(\text { Solidity }+ \text { Arentedimage }+ \text { Perimeter })_{\text {Unsegmentedimage }}
\end{aligned}
$$

Where $\mathrm{S}_{\text {prop }}$ and $\mathrm{O}_{\text {prop }}$ are the region properties of the segmented and unsegmented images, respectively. These metrics are useful for measuring the segmentation accuracy by calculating the region properties of segmented images and its ground truth images.

Solidity is a scalar value which specifies the proportion of the pixels in the convex hull which are also in the region. Area is the actual number of pixels in the region of interest. Convex Area is a Scalar that specifies the number of pixels in Convex Image. Convex Image is a Binary image (logical) that specifies the convex hull, with all pixels within the hull filled in. Perimeter is the distance around the boundary of the region. Region props compute the perimeter by calculating the distance between each adjoining pair of pixels around the border of the region. The results obtained from the proposed metrics for medical images are shown in Table 4. and 5. Themetrics-2 proposed in this paper is an alternative measure to compare the segmentation accuracy since it is more meaningful when compared to method-1. The important properties like solidity, perimeter, and Area can be used. These three values are obtained for segmented and unsegmented (Ground truth) images.

Ground truth image has been formed manually for all the images using Adobe Photoshop. This ground truth image has been formed in discussion with a medical doctor so that the lung and brain is correctly marked with Adobe Photoshop. The percentage of segmentation is relative with respect to the ground truth image

\subsection{Measurement and analysis of over/under segmentation}

To evaluate the segmentation performance, we used two error metrics which are used to measure the oversegmentation and under-segmentation rates. In this proposed segmentation method, we define the over-segmentation of a region of interest as the region volume that is regarded as tissue, and we also define the under-segmentation of the region of interest as the tissue that is regarded as region volume.

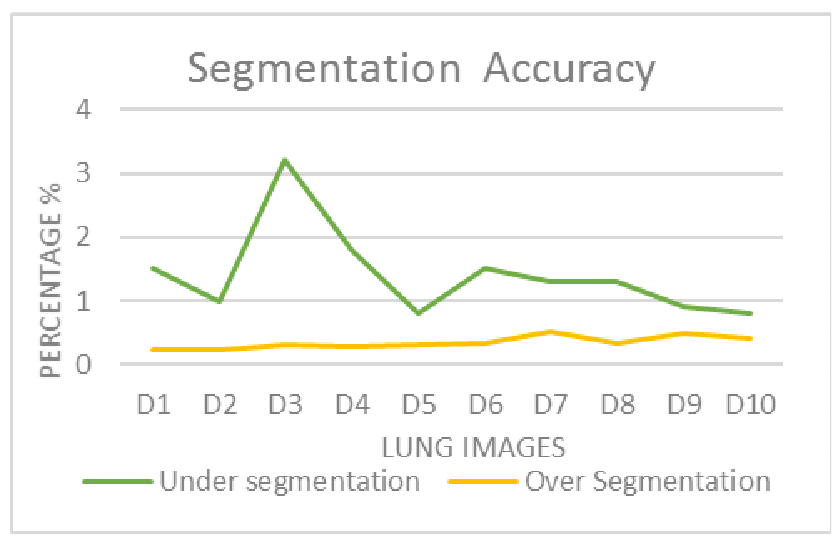

Fig.4. Over segmentation/ under segmentation for lung images.

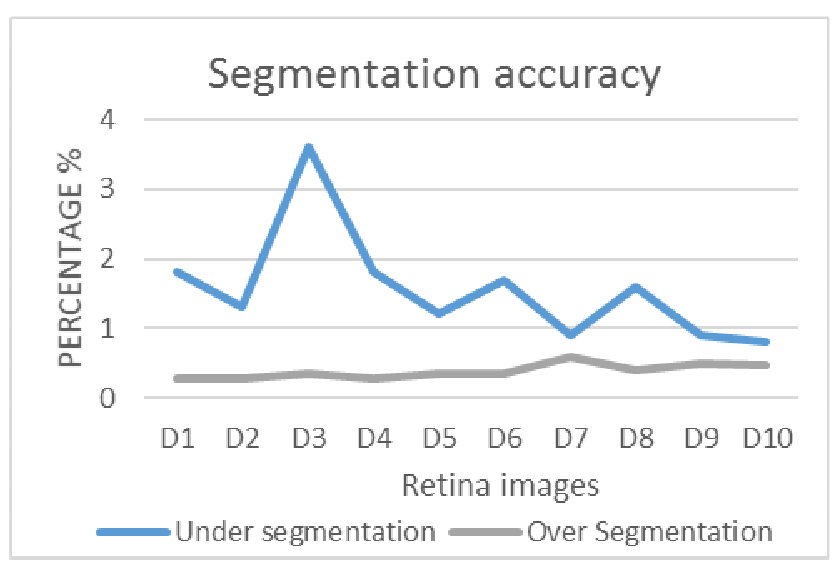

Fig.5. Over segmentation/under segmentation for Retina images.

The over-segmentation and under-segmentation ratios for the 10 lung and retina datasets are shown in Fig.4. and 5. The average over-segmentation volume error per case is $0.32 \%$ and $0.38 \%$ of the entire volume, while the average under-segmentation is $1.39 \%$ and $1.56 \%$, respectively. 
Table 4. Individual segmentation accuracy for lung images using proposed metrics.

\begin{tabular}{|c|c|c|c|c|c|c|}
\hline Image & & Solidity & Area & Perimeter & Total & $\mathrm{A}_{\text {prop }}$ \\
\hline Image 1 & segmented & 0.76535 & 43412 & 1541.4398 & 44954.156335 & \multirow{2}{*}{97.8112} \\
\hline & unsegmented & 0.76535 & 44216 & 1743.4398 & 45960.156335 & \\
\hline \multirow[t]{2}{*}{ Image 2} & Segmented & 0.679 & 37759 & 1832.9068 & 39592.5858 & \multirow{2}{*}{97.5557} \\
\hline & Unsegmented & 0.679 & 38553 & 2030.9068 & 40584.5858 & \\
\hline \multirow[t]{2}{*}{ Image 3} & Segmented & 0.71355 & 31096 & 1307.0235 & 32403.73705 & \multirow{2}{*}{97.2943} \\
\hline & Unsegmented & 0.8376 & 31897 & 1407.0235 & 33304.8611 & \\
\hline \multirow[t]{2}{*}{ Image 4} & Segmented & 0.8376 & 42361 & 1151.918 & 43213.81766 & \multirow{2}{*}{98.4958} \\
\hline & unsegmented & 0.8376 & 42371 & 1501.918 & 43873.7556 & \\
\hline
\end{tabular}

Table 5. Individual segmentation accuracy for retina images using proposed metrics.

\begin{tabular}{|c|c|c|c|c|c|c|}
\hline Image & & Solidity & Area & Perimeter & Total & \multirow{2}{*}{$\mathrm{A}_{\text {prop }}$} \\
\cline { 1 - 6 } Image 1 & segmented & 0.76605 & 32092 & 2411.0335 & 33303.73605 & \multirow{2}{*}{97.5043} \\
\cline { 1 - 6 } & unsegmented & 0.87008 & 33897 & 2407.0235 & 34304.8611 & \\
\hline Image 2 & Segmented & 1.6652 & 40759 & 2832.9568 & 43092.5358 & \multirow{2}{*}{97.6780} \\
\cline { 1 - 6 } & Unsegmented & 0.679 & 38553 & 2030.9068 & 40584.5858 & \\
\hline Image 3 & Segmented & 1.649 & 48135 & 1651.918 & 45213.81766 & \multirow{2}{*}{98.7549} \\
\cline { 1 - 6 } & Unsegmented & 1.6116 & 42371 & 2501.918 & 45873.7556 & \\
\cline { 1 - 5 } Image 4 & Segmented & 1.6553 & 44412 & 2041.4398 & 46454.156335 & \multirow{2}{*}{96.3560} \\
& Unsegmented & 1.6553 & 46216 & 1743.4398 & 49960.156335 & \\
\hline
\end{tabular}

\subsection{Comparison of segmentation accuracy}

Several methods have been shown in the literature for the segmentation of anatomic structures in multiple medical images. Among them, the most commonly used methods are the graph cut method [24], watershed transform [3], shape and location optimization techniques [23] and SpatiallyDivided Probabilistic atlas based method [19]. Table 6 shows the detailed comparison of segmentation accuracy of the proposed method with three existing multi-organ segmentation techniques. From the table, it can be observed that the segmentation accuracy of the proposed methodology is higher when compared to the other existing methods for segmenting organs form the multiple Computed tomography images.

Table 6. Segmentation accuracy for different techniques.

\begin{tabular}{|c|c|c|}
\hline \multicolumn{3}{|c|}{ Segmentation Accuracy } \\
\hline Approaches & Lung image & Retina image \\
\hline watershed transform & $95.305 \%$ & $95.783 \%$ \\
\hline Atlas based method & $96.257 \%$ & $96.827 \%$ \\
\hline Graph cut method & $96.853 \%$ & $96.412 \%$ \\
\hline Proposed Approach & $97.7899 \%$ & $97.588 \%$ \\
\hline
\end{tabular}

\section{CONCLUSIONS}

In this paper, a new fuzzy logic based bitplane thresholding method has been proposed for segmenting the anatomical structures present in multiple medical images effectively. This method overcomes the limitations of existing methods where entire grayscale value of a pixel is considered. Fuzzy logic is used for each bit of a grayscale. Hence, more clarity is obtained in deciding the pixel which requires segmentation. To measure the performance of the proposed segmentation method, new metrics are proposed which measure the segmentation accuracy based on the number of pixels, properties and over/under segmentation ratios. The major contributions of this paper are an algorithm for segmenting multiple CT medical images and a new metrics for the effective measurement of its segmentation accuracy. Future works in this direction can be the proposal of a new technique to analyze the presence of nodules in the segmented regions.

\section{REFERENCES}

[1] Armato, S.G., Giger, M.L., Moran, C.J. (1999). Computerized detection of pulmonary nodules on CT scans. Radio Graphics, 19, 1303-1311.

[2] Hu, S., Huffman, E.A., Reinhardt, J.M. (2001). Automatic lung segmentation for accurate 
quantification of volumetric X-Ray CT images. IEEE Transactions on Medical Imging, 20 (6), 490-498.

[3] Grau, V., Mewes, A.U., Alcañiz, M., Kikinis, R., Warfield, S.K. (2004). Improved watershed transform for medical image segmentation using prior information. IEEE Transactions on Medical Imaging, 23 (4), 447-458.

[4] Pan, Z., Lu, J. (2007). A Bayes-based region-growing algorithm for medical image segmentation. Computing in Science \& Engineering, 9 (4), 32-38.

[5] Mukhopadhyay, S., Chanda, B. (2003). Multiscale morphological segmentation of gray-scale images. IEEE Transactions on Image Processing, 12 (5), 533549.

[6] Maulik, U. (2009). Medical image segmentation using genetic algorithms. IEEE Transactions on Information Technology in Biomedicine, 13 (2), 166-173.

[7] Holtzman-Gazit, M., Kimmel, R., Peled, N., Goldsher, D. (2006). Segmentation of thin structures in volumetric medical images. IEEE Transactions on Image Processing, 15 (2), 354-363.

[8] Antonelli, M., Lazzerini, B., Marcelloni, F. (2005). Segmentation and reconstruction of the lung volume in CT images. In ACM Symposium on Applied Computing, 13-17 March 2005. ACM, 255-259.

[9] Pu, J., Roos, J., Yi, C.A., Napel, S., Rubin, G.D., Paik, D.S. (2008). Adaptive border marching algorithm: Automatic lung segmentation on chest $\mathrm{CT}$ images. Computerized Medical Imaging and Graphics, 32 (6), 452-462.

[10] Prasad, M.N., Brown, M.S., Ahmad, S., Abtin, F., Allen, J., Da Costa, I., Kim, H.J., McNitt-Gray, M.F., Goldin, J.G. (2008). Automatic segmentation of lung parenchyma in the presence of diseases based on curvature of ribs. Academic Radiology, 15 (9), 1173 1180.

[11] Meng, X., Qiang, Y., Zhu, S., Fuhrman, C., Siegfried, J.M., Pu, J. (2012) Illustration of the obstacles in computerized lung segmentation using examples. Medical Physics, 39 (8), 4984-4991.

[12] Xu, L., Luo, S. (2010). A novel method for blood vessel detection from retinal images. BioMedical Engineering OnLine, 9 (1), 2-10.

[13] Gunadi, W.N., Shamsuddin, S.M., Alias, R.A., Sap, M.N. (2003). Selection of defuzzification method to obtain crisp value for representing uncertain data in a modified sweep algorithm. Journal of Computer Science \& Technology, 3 (2), 22-28.

[14] Lopes, N.V., Mogadouro do Couto, P.A., Bustince, H., Melo-Pinto, P. (2010). Automatic histogram threshold using fuzzy measures. IEEE Transactions on Image Processing, 19 (1), 199-204.

[15] Jain, A.K. (1989). Fundamentals of Digital Image Processing. Prentice-Hall.
[16] Beevi, Z., Sathik, M. (2012). A robust segmentation approach for noisy medical images using fuzzy clustering with spatial probability. The International Arab Journal of Information Technology, 9 (1), 74-83.

[17] Kalender, W.A., Fichte, H., Bautz, W., Skalej, M. (1991). Semiautomatic evaluation procedures for quantitative CT of the lung. Journal of Computer Assisted Tomography, 15, 248-255.

[18] Xie, X.L., Beni, G.A. (1991). Validity measure for fuzzy clustering. IEEE Transactions on Pattern Analysis and Machine Intelligence, 3, 841-846.

[19] Chu, C., Oda, M., Kitasaka, T., Misawa, K., Fujiwara, M., Hayashi, Y., Nimura, Y., Rueckert, D., Mori, K. (2013) Multi-organ segmentation based on spatially-divided probabilistic atlas from 3D abdominal CT images. In Medical Image Computing and Computer-Assisted Intervention. Springer, LNCS 8150, 165-172.

[20] Suzuki, M., Linguraru, M.G., Okada, K. (2012) Multiorgan segmentation with missing organs in abdominal CT images. In Medical Image Computing and Computer-Assisted Intervention. Springer, LNCS 7512, 418-425.

[21] Xinge You, Qinmu Peng, Yuan Yuan, Yiu-ming Cheung, Jiajia Lei. (2011). Segmentation of retinal blood vessels using the radial projection and semisupervised approach. Pattern Recognition, 44 (10-11), 2314-2324.

[22] Smirg, O., Liberda, O., Smekal, Z., Sprlakova-Pukova, A. (2012). MRI slice segmentation and 3D modelling of temporomandibular joint measured by microscopic coil. Measurement Science Review, 12 (3), 74-81.

[23] Linguraru, M.G., Pura, J.A., Chowdhury, A.S., Summers, R.M. (2010). Multi-organ segmentation from multi-phase abdominal CT via 4D graphs using enhancement, shape and location optimization. In Medical Image Computing and Computer-Assisted Intervention. Springer, LNCS 6363, 89-96.

[24] Zheng, Y., Stambolian, D., O’Brien, J., Gee, J.C. (2013). Optic disc and cup segmentation from color fundus photograph using graph cut with priors. In Medical Image Computing and Computer-Assisted Intervention. Springer, LNCS 8150, 75-82.

[25] Marin, D., Aquino, A., Gegundez-Arias, M.E., Bravo, J.M. (2011). A new supervised method for blood vessel segmentation in retinal images by using graylevel and moment invariants-based features. IEEE Transactions on Medical Imaging, 30 (1), 146-158.

[26] Fraz, M.M., Remagnino, P., Hoppe, A., Uyyanonvara, B., Rudnicka, A.R., Owen, C.G., Barman, S.A. (2012). An ensemble classification-based approach applied to retinal blood vessel segmentation. IEEE Transactions on Biomedical Engineering, 59 (9), 2538-2548.

Received July 22, 2013 Accepted April 11, 2014. 